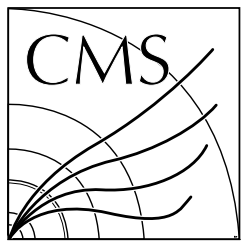

The Compact Muon Solenoid Experiment
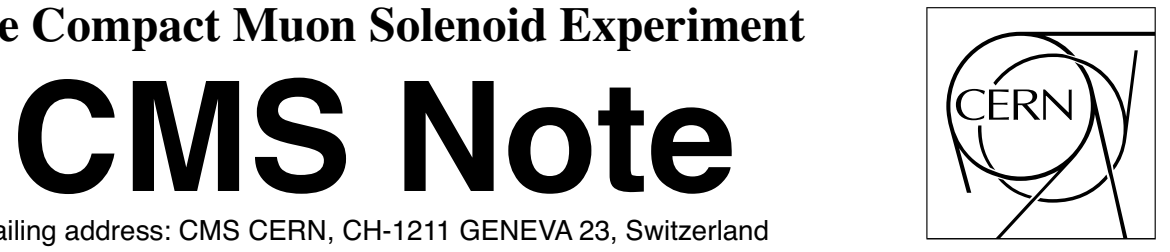

18 June 2017 (v4, 01 September 2017)

\title{
The drift velocity monitoring system of the CMS barrel muon chambers
}

\author{
Georg Altenhöfer ${ }^{1)}$, Thomas Hebbeker, Carsten Heidemann, Hans Reithler, Lars Sonnenschein ${ }^{2)}$ and Daniel \\ Teyssier
}

III. Phys. Institute A, RWTH Aachen University

\begin{abstract}
The drift velocity is a key parameter of drift chambers. Its value depends on several parameters: electric field, pressure, temperature, gas mixture, and contamination, for example, by ambient air. A dedicated Velocity Drift Chamber (VDC) with 1-L volume has been built at the III. Phys. Institute A, RWTH Aachen, in order to monitor the drift velocity of all CMS barrel muon Drift Tube chambers. A system of six VDCs was installed at CMS and has been running since January 2011. We present the VDC monitoring system, its principle of operation, and measurements performed.
\end{abstract}

\footnotetext{
1) Now at Forschungszentrum Jülich, Jülich, Germany

2) Now at ADU cologne GmbH, Cologne, Germany
} 


\section{Introduction}

The Compact Muon Solenoid (CMS) [1] experiment is a multipurpose detector at the Large Hadron Collider (LHC) at CERN capable of triggering, measuring, and identifying several different objects (in particular electrons, photons, jets, and muons). The aim of the CMS muon system is to trigger on the muon candidates and measure their momentum [2][3]. The barrel muon chambers are made of 250 Drift Tube (DT) chambers organized into five wheels. Since the drift time serves as a measure of the track position, a precise knowledge of the drift velocity $\left(v_{\text {drift }}\right)$ is required in the DT chambers [1][2] for muon track reconstruction. The hit position is determined using the nominal $v_{\text {drift }}$ and the measured drift time, assuming a linear dependency. Several parameters can influence the $v_{\text {drift }}$ value: the electric field, pressure, temperature, gas mixture, and the level of contamination by $\mathrm{O}_{2}$ and $\mathrm{N}_{2}$ from ambient air. A set of six Velocity Drift monitoring Chambers (VDC) was constructed in order to monitor this key parameter in all 250 DT chambers. The VDC system has been developed and built at the RWTH Aachen University, III. Phys. Institute A (see, for example, [4][5]). It is a further development of a similar monitoring chamber designed for the L3 experiment [6]. In these chambers, two narrow beams of beta particles from ${ }^{90} \mathrm{Sr}$ radioactive sources are used. The same principle has also been applied for applications at lower E-field in [8][9], and at higher E-field in [10]. Alternatively, devices based on extraction of electrons from a cathode by laser beams [7] and on gas ionization by laser beams [11] have been reported. Common to all these $v_{\text {drift }}$-chambers is the use of a homogeneous electric field in the region between the two ionizing beams, and being insensitive to the unavoidable non-linearities near the anode wire by subtracting measurements from two different distances. Equipped with an adjustable electric field, the VDCs are also well suited for the study of gas properties. The VDCs installed in CMS are similar to previous ones used in the L3 experiment but operate at higher electric field and are more serviceable. The complete VDC system has been operating since 2011 in the CMS underground cavern to perform this monitoring.

\section{Drift velocity monitoring}

\subsection{Precision requirements}

The drift tubes used in the barrel muon chambers in CMS consist of rectangular cells with a pitch of $42 \mathrm{~mm}$, a height of $13 \mathrm{~mm}$, and a 20.3-mm effective travel length. They all have cathodes, strips, and an anode wire located in the middle of the gas volume. The average electric field inside is about $200 \mathrm{~V} / \mathrm{mm}$. The hit resolution should be in the range between 200 and $250 \mu \mathrm{m}$ in order to get the expected muon $\mathrm{p}_{\mathrm{T}}$ resolution (better than $1 \%$ for $\mathrm{p}_{\mathrm{T}}$ below $100 \mathrm{GeV}$ ). It implies that the accuracy of $v_{\text {drift }}$ should be better than the ratio of the hit resolution over the half-width of the cell, which means lower than $1 \%$. This is an upper limit for the resolution, for the longest possible path to the anode wire. The nominal $v_{\text {drift }}$ is around $54.5 \mu \mathrm{m} / \mathrm{ns}$ and thus the total uncertainty from the VDC measurement should not exceed $0.5 \mu \mathrm{m} / \mathrm{ns}$. For monitoring purposes, we attempt to recognize variations as small as around $0.1 \mu \mathrm{m} / \mathrm{ns}$.

In a single barrel muon chamber at the common gas return line of the 250 installed chambers, the effect is diluted in the same proportion. To be sensitive to a similar variation of $v_{\text {drift }}$ in a single chamber would require $v_{d r i f t}$ to be measured with 250 times better resolution, which would still not allow the identification of the chamber concerned. Therefore, to ensure the same sensitivity at the level of a single chamber, the VDC system is designed to allow sampling from every barrel muon chamber.

\subsection{Parameters influencing the drift velocity}

Several parameters can influence the value of $v_{\text {drift }}$ in the barrel muon chambers. First of all, in each drift cell of the CMS DT system, electrodes at three different voltages are used as a simple method to shape the electric field, such that most of the drift lines do not run straight to the anode and the electric field varies along the drift lines. For those DT chambers that are exposed to a significant fraction of the magnetic field of the CMS solenoid, the drift lines will be further affected and the effective drift velocity will appear to be lower. Pressure and temperature also affect $v_{d r i f t}$, increasing with temperature, and decreasing with pressure. Pressure, temperature and electric field are monitored by measuring the high voltage, the gas pressure, and the temperature in the DT chambers.

To a good approximation, $v_{d r i f t}$ should depend on the electric field divided by the gas density; hence for an electric field $E$, pressure $p$, and temperature $T$ (in Kelvin), $v_{\text {drift }}$ is a function of $E /(p / T)$. This permits the use of data for $1000 \mathrm{mbar}$ and $20^{\circ} \mathrm{C}$, while accounting for small deviations from these values by scaling $E$ accordingly.

Two other parameters are linked to the gas quality: the nominal composition of the gas mixture and a possible contamination with other gas impurities. A precise analysis of the actual gas composition, besides being very 
demanding, is also difficult to convert into a drift velocity. The aim of the VDC system is to monitor the drift velocity by a direct measurement under defined conditions of pressure, temperature, and electric field.

\subsubsection{Gas composition}

The nominal gas used in the DT system is a mixture of $\mathrm{Ar}(85 \%)$ and $\mathrm{CO}_{2}(15 \%)$. The $v_{\text {drift }}$ value strongly depends on the gas composition. The expectation values for $v_{\text {drift }}$ for different gas mixtures and electric fields were derived using the software package Magboltz [12]. Figure 1 shows a simulation with Magboltz of $v_{d r i f t}$ curves as a function of the electric field $\mathrm{E}$ for modest variations of the mixing ratio of $\mathrm{Ar}$ and $\mathrm{CO}_{2}$. The width of each curve is the error estimation of Magboltz. Even a slight deviation by $1 \%$ of the components can induce a $2 \%$ change of the $v_{\text {drift }}$ value, which is a deviation too large for the DT system.

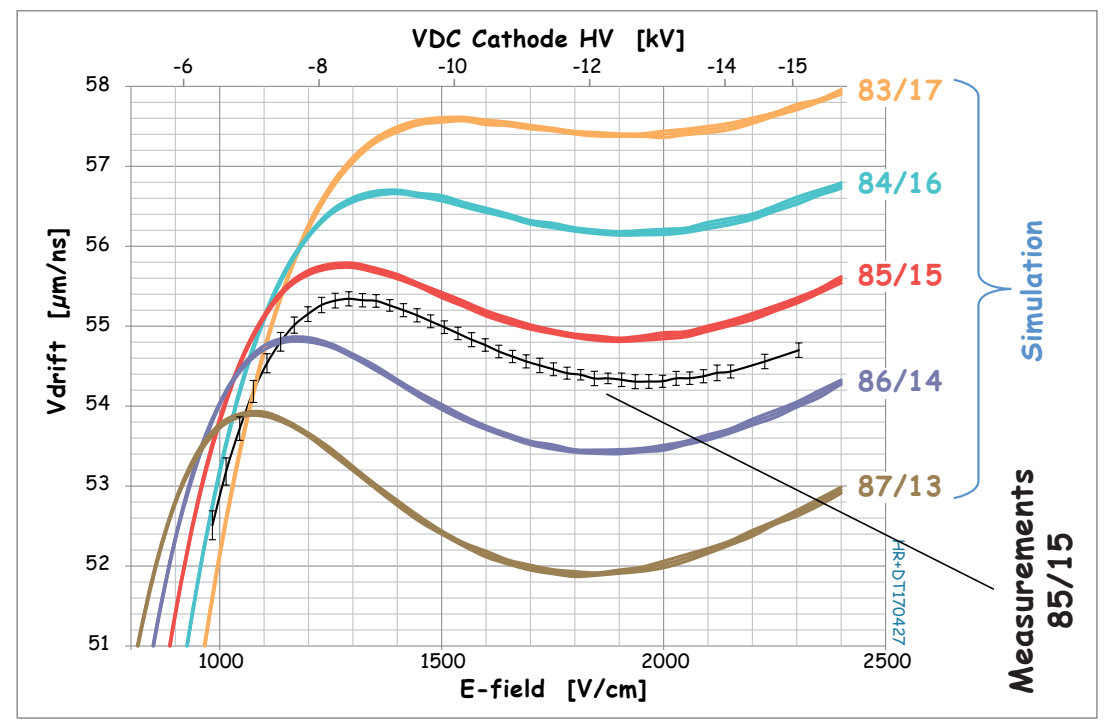

Figure 1: Simulation of the drift velocity $v_{\text {drift }}$ using different gas compositions around the nominal mixture of $85 \% \mathrm{Ar}$ and $15 \% \mathrm{CO}_{2}$, without impurities. The measurements by VDCs are also shown.

The measurement shown is the average from four VDCs, from runs at +1750 and $+1800 \mathrm{~V}$ anode voltage, i.e., from eight measurements, with each point measured for $30 \mathrm{~min}$, using typical anode settings, to avoid statistical fluctuationsi (usual monitoring with VDCs is done for $10 \mathrm{~min}$ per point and repeated six times). The statistical uncertainty for each point is around $0.05 \mu \mathrm{m} / \mathrm{ns}$; thus it is below $0.02 \mu \mathrm{m} / \mathrm{ns}$ for the average over the eight measurements. The observed standard deviation of the measurements, indicated by the size of the markers in the figure, amounts to $0.1 \mu \mathrm{m} / \mathrm{ns}$ and is dominated by the systematic difference among the four VDCs used in these runs. Indeed, the measurements' eight curves are smooth but slightly shifted with respect to each other. The observed small fluctuations are evidence of the ability of the VDC to recognize $v_{\text {drift }}$ variations of $0.1 \mu \mathrm{m} / \mathrm{ns}$. The expected uncertainties of the Magboltz simulation are even smaller than those of the measurement, while the knowledge of $(15 \pm 0.075) \%$ for the $\mathrm{CO}_{2}$ admixture of the pure gas used ${ }^{1)}$ corresponds to an uncertainty of $\pm 0.1 \mu \mathrm{m} / \mathrm{ns}$ for $v_{\text {drift }}$. With these uncertainties, the simulation and measurement shown in the figure do not fully agree. The origin of this difference of up to $0.4 \mu \mathrm{m} / \mathrm{ns}(0.7 \%)$ could not be identified, but we can state that from Fig. 1, the measured absolute values of $v_{\text {drift }}$ are confirmed to $0.7 \%$. Earlier measurements [10] of the same gas mixture, also covering the range of the electric fields of the present work, agree with the VDC measurements within the estimated uncertainties.

\subsubsection{Gas contamination}

Because the hermeticity of the entire gas system and muon chambers cannot be perfect, there could be some contamination, in particular by ambient air. The presence of some impurity in the main gas mixture could also affect the drift velocity. To avoid a large contamination in case of a leak, the gas system of the muon chambers is

1) Analysis grade, quality 50, "Diamond" class from Carbagas A.G. : www.carbagas.com. Binary mixture with $85 / 15 \mathrm{Ar} / \mathrm{CO}_{2}$ certified to a best measurement of $\pm 0.5 \%$ relative to the $15 \%$, i.e., a mixture certified to contain $(15 \pm 0.075) \% \mathrm{CO}_{2}$. 
operated at a slight overpressure of 3 mbar. As shown in Fig. 2, from simulation with Magboltz [12], the expected impact of an $\mathrm{N}_{2}$ contamination of $500 \mathrm{ppm}$, for example, on $v_{d r i f t}$ is about $0.4 \mu \mathrm{m} / \mathrm{ns}$, which is easily measurable by the VDC. Contamination by $\mathrm{N}_{2}$ tends to increase the value of $v_{d r i f t}$. Humidity has a similar but smaller impact; $500 \mathrm{ppm} \mathrm{H}_{2} \mathrm{O}$ leads to an increase of less than $0.1 \mu \mathrm{m} / \mathrm{ns}$. Contamination by $\mathrm{O}_{2}$ has about a six times smaller impact on $v_{\text {drift }}$ than contamination with $\mathrm{N}_{2}$, but $\mathrm{O}_{2}$ tends to absorb the drifting electrons and hence to reduce the signal amplitude. The presence of any other impurity is also accounted for by the VDC data, since it measures $v_{\text {drift }}$ directly.

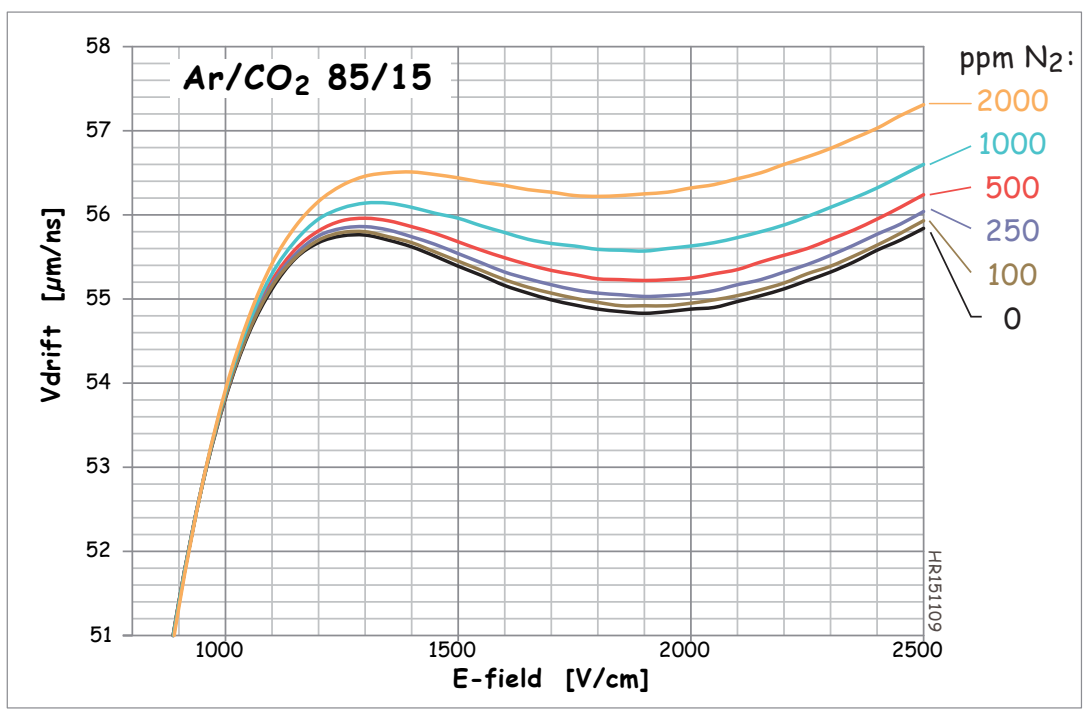

Figure 2: Simulation of $v_{d r i f t}$ for a $85 / 15 \mathrm{Ar} / \mathrm{CO}_{2}$ gas mixture with several levels of $\mathrm{N}_{2}$ contamination.

\section{Description of the VDC}

\subsection{Working principle}

The Velocity Drift Chamber (VDC) is a small chamber with 1-L volume dedicated to monitoring the $v_{\text {drift }}$, with a faster exchange of gas compared to the targeted DT stations. The E-field inside the VDC is homogeneous and kept constant, which allows a direct $v_{\text {drift }}$ measurement at a given E-field to be accomplished by measuring the difference between arrival times of electrons from two positions. The E-field is adjustable, which permits the measurement of $v_{\text {drift }}$ as a function of the E-field to reproduce the DT conditions, and gas samples from the DT chambers are piped to the VDC monitoring system to check for gas anomalies. Figure 3 shows the working principle of a VDC.

\subsection{Layout}

The system consists of a chamber equipped with an anode wire of $30-\mu \mathrm{m}$ diameter with high voltage up to $+2000 \mathrm{~V}$, a flat cathode with high voltage up to $-15000 \mathrm{~V}$, and field shaping electrodes. Two electron beams from ${ }^{90} \mathrm{Sr}$ sources cross a sensitive region between the anode and cathode. The sensitive region is characterized by a highly uniform electric field. The arrangement of the field electrodes is optimized to ensure a uniformity better than $0.4 \%$ in this region. The electrons from the radioactive sources are detected by a scintillating fiber and two silicon photomultipliers (SiPM) placed at both ends of the fiber and matching their cross section of $1 \mathrm{~mm}^{2}$. The coincidence between the two SiPM signals serves as trigger and provides a time reference for the passage of an electron from the radioactive sources. The scintillating fiber is U-shaped, with the two parallel branches of the "U" located in front of the exit slits of the electrons from the radioactive sources. They are thus parallel to both the anode wire and field-shaping electrodes. The width of the fiber $(1 \mathrm{~mm})$ and the distance between the two slits $(48 \mathrm{~mm})$ define the geometry for the measurement of $v_{\text {drift }}$. The drift times of negative charges produced by the two beams reaching the anode wire are recorded with respect to the trigger signal time. The average drift time between both parallel beams, separated by $48 \mathrm{~mm}$, is then extracted from the two peaks visible in the overall time distribution. This time difference is converted into a drift velocity. 

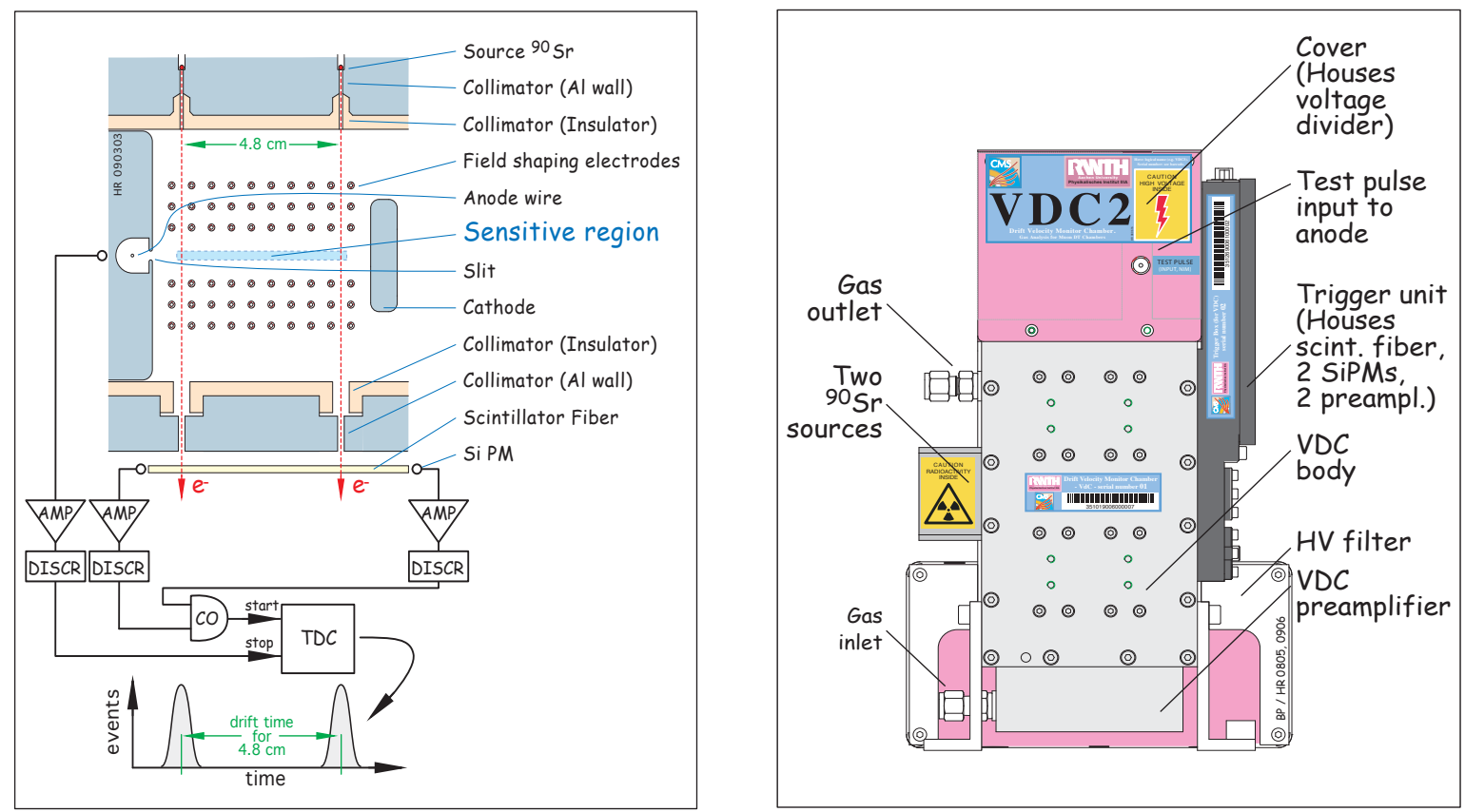

Figure 3: Left: Working principle and geometry of a Velocity Drift Chamber (VDC). The region where the electric field should be adjustable while staying very homogeneous is labelled the sensitive region. Right: Front view of a VDC with its main components. The anode wire and the shaping electrodes are oriented vertically in this view.

The electric field of the VDC can be varied in a wide range without losing its homogeneity. The VDC measures the drift velocity directly for a given electric field, while in the DTs the determined drift velocity is an effective parameter parameter that depends on, besides the gas admixture, the electric field, path of the electrons drifting to the anode, software selection, and fit algorithms. The drift velocity measured with the VDC can be exploited to check the gas admixture and determine the level of contamination by other gases of a given muon station.

\subsection{The 6-VDC system}

The complete VDC system is housed in three racks (Fig. 4).

The rack at the left in Fig. 4 contains the High Voltage (HV) supply with three multichannel HV modules ${ }^{2)}$. The rack at the center contains VME logic modules, NIM discriminator modules and a readout PC (Linux server). The VME modules for the readout of the drift time include the following:

- fanout and coincidence modules

- modules to link NIM logic to a Time to Digital Converter (TDC):

1. NIM-ECL Converter, which is AC/DC coupled and displays activity, to convert 32 NIM signals to complementary ECL ones

2. ECL adapter to route ECL signals to the five connectors on the TDC

- TDC module

- input register, which, for instance, senses an external power-off alarm and switches off the system

- output register, which, for example, activates test pulses

- VME scalers to count the signals independently from the restricted time window of the TDC

- VME controller with a USB link to the PC.

2) SY1527 crate, from CAEN. 


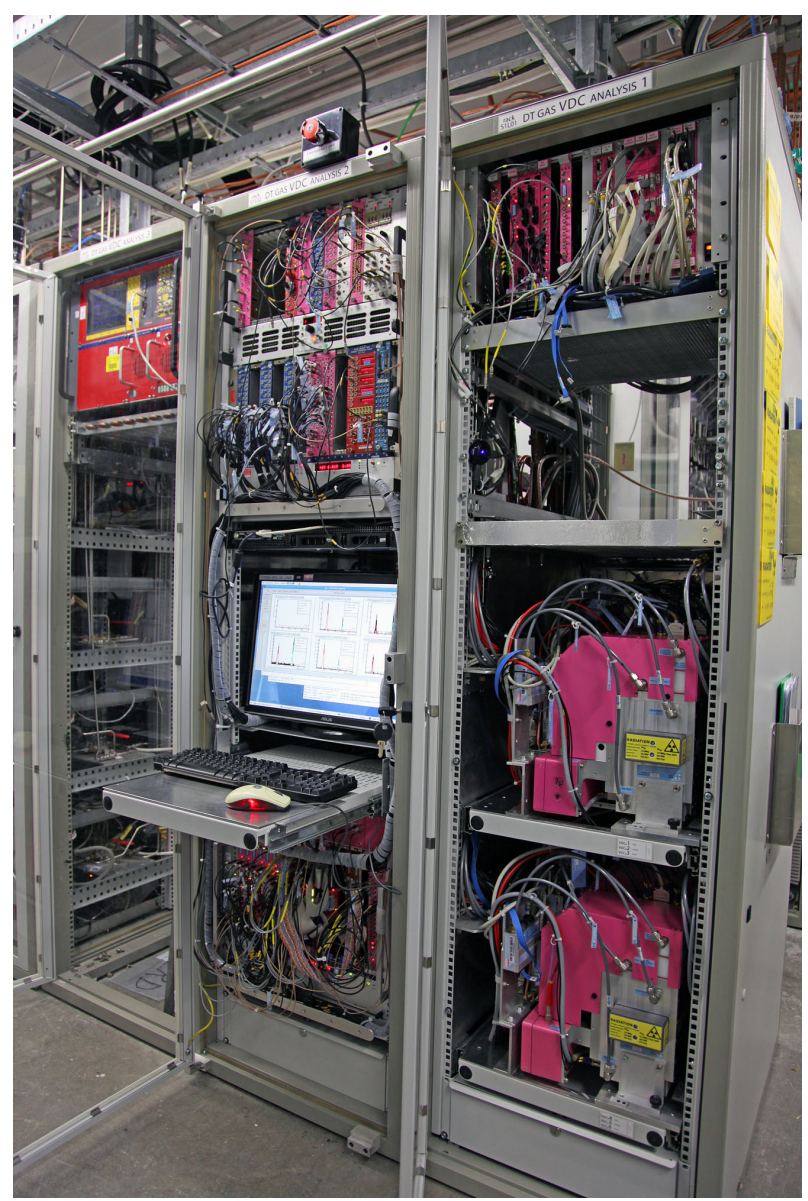

Figure 4: Overview picture of the VDC system housed in three racks at Point 5 (CMS) of the LHC at CERN. Two VDCs are visible on the right. Groups of three VDCs are mounted in a drawer that can be pulled out for maintenance work without disconnecting them. 
The rack at the right contains six VDCs (one for each wheel plus a spare), pressure controllers and sensors, a trigger unit on each VDC, and a crate with dedicated VME modules. The six VDCs are arranged in groups of three on sliding trays to ensure maintenance access without interrupting ongoing operation. The VME modules include the following:

- Low Voltage (LV) module for the VDC preamplifier and temperature reading

- Supply Tri-Box module to provide LV for the trigger box preamplifiers to route adjustable voltage to the SiPM's, and to read temperatures

- Fan Out $($ FO $6 \times 4)$ module for NIM signals with four groups

- Pressure Link module to provide LV for pressure and temperature sensors located in the gas manifold, to route the pressure signal to the ADC card plugged into a linux PC

- pulser to provide test pulses with two different levels and eight outputs programmable via USB

- Valve Readout module to provide heater pulse and reading of temperature sensors via USB, including the temperatures from other modules.

\section{Environment of the VDC}

Although basically a small volume single cell drift chamber, nevertheless, each VDC requires the same complete environment as does a large, full-fledged detector, both in terms of hardware and software. This concerns trigger, supplies, gas distribution, gas flow and pressure regulation, safety alarms, readout and time digitization of trigger and VDC signals, local monitoring and logging by software, as well as communication with the general CMS software system. The trigger unit is described in chapter 3 .

\subsection{Hardware Environment}

The high voltage needs for each VDC are about -9 to $-15 \mathrm{kV}$ for the cathode, up to $+2 \mathrm{kV}$ for the anode, and up to $+70 \mathrm{~V}$ for the two light detectors (SiPM) of the trigger unit. For the voltages to be individually adjustable for each of the six VDCs, high voltage modules with multiple channels were used; for each of the three voltages one module is sufficient and they are housed in a single crate ${ }^{3)}$. The readout logic consists of discriminator, fanout, coincidence, timing unit modules ${ }^{4)}$ in NIM and VME format, working with NIM signals. At the end of the chain, using a NIM to ECL converter, the signals are fed to VME scaler and TDC units, which are read out via a USB interface module ${ }^{5)}$. The TDC is a multihit digital unit with $0.7 \mathrm{~ns}$ resolution ${ }^{6}$. For each VDC the arrival times of signals from the anode, from both SiPMs, and from the coincidence between the two SiPM signals are recorded, thus requiring $24 \mathrm{TDC}$ channels in total. The time window for each event is $2.5 \mu$ s wide and starts about $200 \mathrm{~ns}$ before the arrival of the global trigger. The trigger for each VDC is given by the coincidence of its two SiPM signals, while the OR of these triggers forms the global trigger, which is also recorded in the TDC. The event record contains the data from all TDC channels, i.e., from all VDCs; the VDC that fired is readily identified as the VDC that has a trigger entry in the TDC, and the time of its trigger signal is the same as the time of the global trigger. This is independent from the presence of an anode signal. A set of scaler channels serves also to monitor all the signal rates outside the short time windows following the trigger pulses, therefore complementing the information available via the TDC channels.

Five pipes for the gas sampling from the large DT muon chambers arrive at the VDC rack. Each of these sampling lines is connected to a remotely controlled multiway valve located close to a group of 50 muon chambers. This permits sampling from a single muon chamber outlet or from the common inlet line to this group of muon chambers. The DT chambers are operated with a large volume of $85 / 15 \mathrm{Ar} / \mathrm{CO}_{2}$ mixture (this gas is run in a closed circuit with a purifier and a continuous substitution of about $15 \%$ of the gas volume per cycle): this is the main gas to be analyzed by the VDCs. Further gas lines linked to the VDC rack are from gas bottles containing

\footnotetext{
3) A1526N, A1821P, A1510, in crate SY1527, from CAEN.

4) Built at III. Phys. Institute A, RWTH Aachen.

5) VM-USB, from WIENER.

6) V767, from CAEN.
} 
analysis-grade premixed gas for (inter-)calibration checks. In addition, gas bottles with well defined amounts of $\mathrm{N}_{2}$ and $\mathrm{O}_{2}$ impurities are available. In order to ensure a constant and well-defined gas flow and pressure inside the VDC, a mass-flow controller upstream and a pressure controller downstream the VDC are installed ${ }^{7)}$. Operation is at $5 \mathrm{~L} / \mathrm{h}$ and $1000 \mathrm{mbar}$.

Special care was devoted to satisfying the requirements of intercalibration among the VDCs and of uninterrupted operation of the system in case of a VDC failure to avoid long down times required for repair and after opening the gas circuit. For this, the piping between the five sampling lines from the muon chambers and the VDCs is organized as a matrix (Fig. 5). The gas from the sources can readily be routed to any of the VDCs at any time. A sixth VDC is installed as a spare unit for the five sampling lines from the muon chambers. Switching over to the spare VDC in case of a failure or for maintenance is done readily by simply turning its valve. Moreover, while all VDCs are operational the spare VDC can run in parallel to any other VDC to cross-check the measurement. Further extensions to this gas matrix are connections to gas lines from bottles of reference gas, as well as sampling from the global gas system inlet and outlet. The sixth VDC can be used to measure one of these gas lines while it is not needed for its main task as a spare unit. Valves are also installed at the exhaust of each VDC to permit checking of the gas tightness of the system. Regular valves for hand operation were chosen for the routing in the gas matrix, 45 in total. To ensure correct knowledge and recording of the actual assignment of gas sources to VDCs, the position of the handle of each valve is read out every ten minutes. A small bulb under the handle is powered by an electric pulse of a few seconds. Two temperature sensors on a fixed printed circuit board underneath the handle, one at the open position and one at the closed position of the handle, can detect whether the valve is open, closed, or in an intermediate position. Temperature sensors with integrated digitizer ${ }^{8)}$ with a resolution of $0.1^{\circ} \mathrm{C}$ are read via a common bus for each VDC. A temperature increase well above $1^{\circ} \mathrm{C}$ is required to identify the position of the valve handle.

The temperature sensors of the valve readout also measure the rack temperature in the intervals between the heating pulses. Further temperature sensors are located inside the VDCs, the trigger units, and at some other places of the rack. An independent set of pressure sensors ${ }^{9}$, located close to the VDC, monitors the performance of the pressure regulator. They are read out by a 16-bit ADC.

Monitoring of the oxygen and the humidity content of the sampled gas is done with commercial units located in a nearby rack.

A second PC with installed software is present for a rapid swap in case of failure.

\subsection{Software Environment}

The VDC software runs on a local PC in a fully autonomous way. Communication with other components of the gas system of the muon chambers and with the detector control system of CMS is carried out via an infrastructure developed at CERN called Data Interchange Protocol (DIP ${ }^{10)}$ ) that permits the exchange of small amounts of real-time data in a heterogeneous system. On the VDC PC the software consists of many processes running independently. Information exchange between the processes and their control is mainly done via text files. A background process watches the activity of all components of the VDC software, starts them and also restarts them in case of a stop or crash. Data on gas pressure, flow, temperature, gas circuit status, TDC data, high voltage including warnings, rates, hardware configuration, etc. are logged for every measurement. To ease the routine task of checking the data, a daily summary report is generated, stored, and sent by email to the maintainers. Warnings, for instance, of an overcurrent, are sent as automatic messages to the operator and are logged. This flexible organization of the software permits changes to be done in the gas circuit and the maintenance work on a VDC to be accomplished while the others are in normal data acquisition mode. We can also inspect the software operation during data taking without interruption or interference with the data taking.

\footnotetext{
7) Series HI-TEC, from Bronkhorst.

8) DS1820, from Maxim.

9) MPX5100, MPX5500, MPXHZ6130A, from Freescale.

10) https://j2eeps.cern.ch/wikis/display/EN/DIP+and+DIM
} 


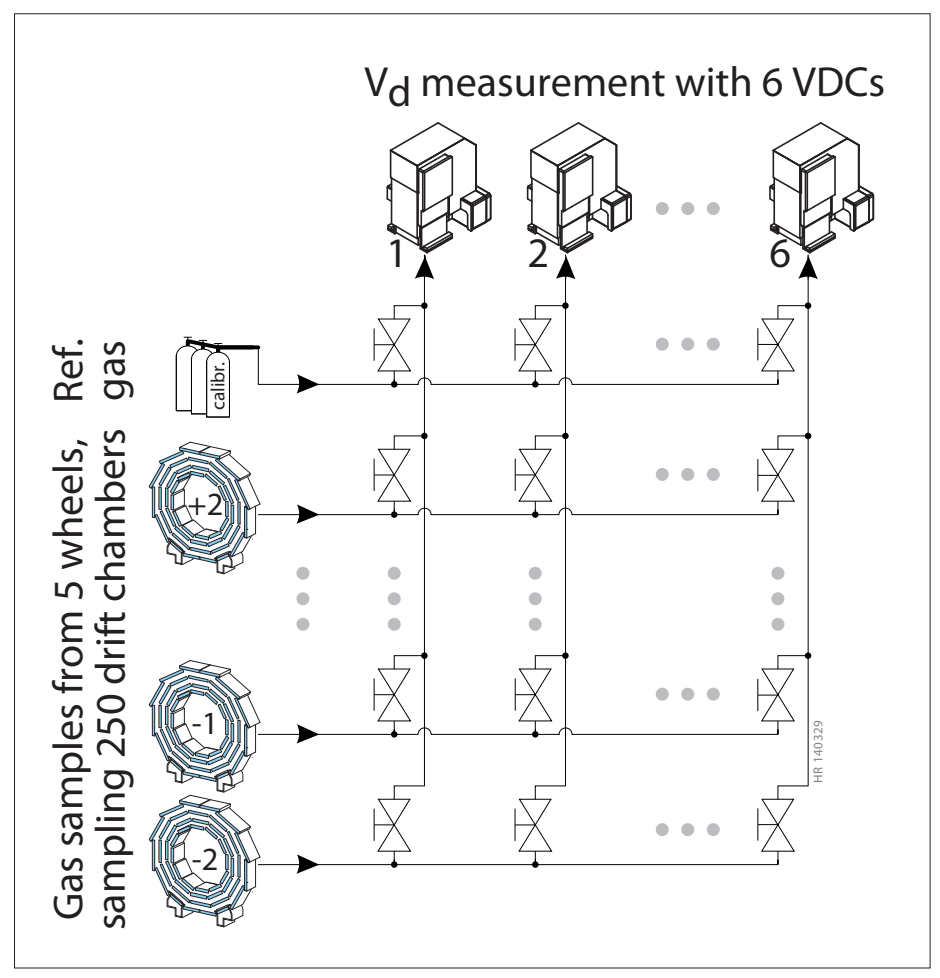

Figure 5: The gas samples from the five groups of DT chambers are routed to the VDC inlet of six VDCs in a flexible selectable way. The sixth VDC serves as a spare for any of the other five VDCs. This matrix arrangement permits the intercalibration of the VDCs to be performed by feeding them with the same gas. A further connection to gas bottles with reference gas is also provided. 


\section{Commissioning and performance}

\subsection{First tests and commissioning}

The full 6-VDC system is operating in the CMS underground cavern since January 2011. Figure 6 shows an example of a drift time spectrum obtained over a 10-min measurement at $-13 \mathrm{kV}$ and 1000 mbar pressure. The uncertainties shown include the statistical error and a position uncertainty of $28 \mu \mathrm{m}$ on the $48 \mathrm{~mm}$ distance. For the usual measurements of $10 \mathrm{~min}$, the statistical uncertainty is comparable to the systematic uncertainty. Possible distortions caused by occasional afterpulsing are avoided by taking the first anode hit of each event. The uncertainty in all cases is already small enough to meet the required precision of $0.5 \mu \mathrm{m} / \mathrm{ns}$. Table 1 summarises the $v_{d r i f t}$ values calculated from the drift time spectra of the same 10-min run, with their corresponding uncertainties. The average $v_{\text {drift }}$ value of $54.8 \mu \mathrm{m} / \mathrm{ns}$ is consistent with the expectation for an $85 / 15 \mathrm{Ar} / \mathrm{CO}_{2}$ mixture containing in addition around $100 \mathrm{ppm}$ of $\mathrm{O}_{2}$, about $400 \mathrm{ppm}$ of $\mathrm{N}_{2}$, and $500 \mathrm{ppm}$ of $\mathrm{H}_{2} \mathrm{O}$. Some constant differences between the $v_{d r i f t}$ values observed in the six VDCs can be explained by the systematic uncertainties.

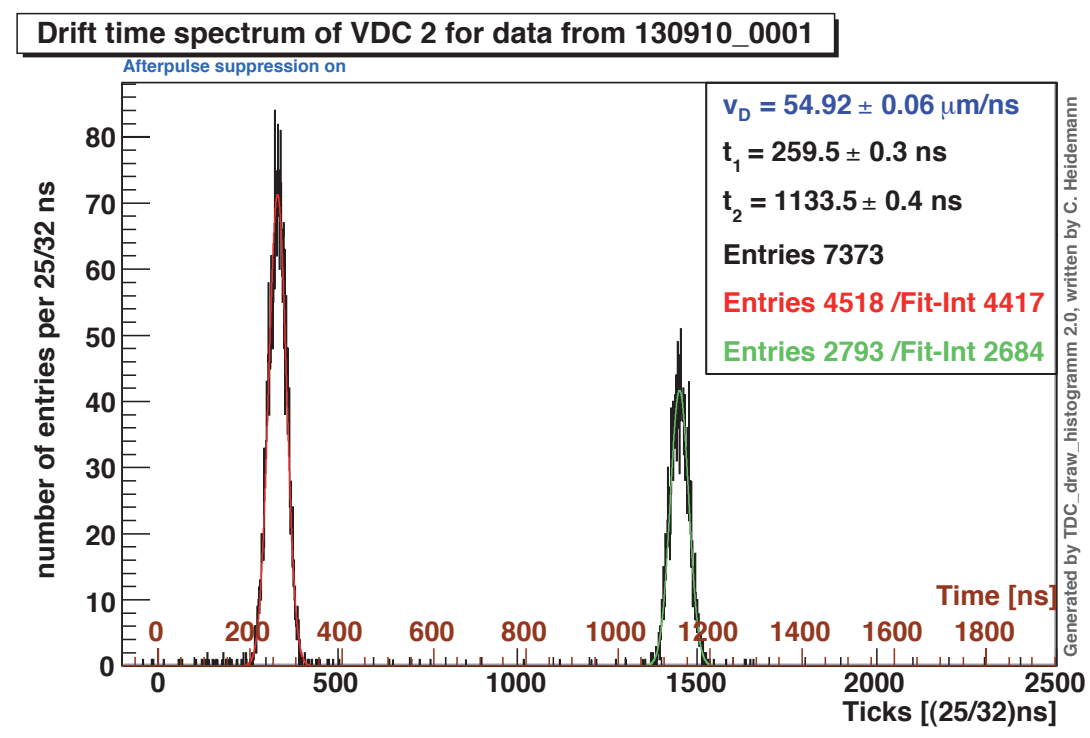

Figure 6: Example of a drift time distribution from a 10-min run, as obtained during 2013 data taking operations, with the fitted curves superimposed. The derived $v_{\text {drift }}$ and its uncertainty are also indicated.

\begin{tabular}{|c|c|c|}
\hline & $v_{\text {drift }}[\mu \mathrm{m} / \mathrm{ns}]$ & Uncertainty $[\mu \mathrm{m} / \mathrm{ns}]$ \\
\hline VDC1 & 54.67 & 0.05 \\
VDC2 & 54.92 & 0.06 \\
VDC3 & 54.71 & 0.09 \\
VDC4 & 54.81 & 0.05 \\
VDC5 & 54.77 & 0.06 \\
VDC6 & 54.85 & 0.05 \\
\hline
\end{tabular}

Table 1: Measured $v_{d r i f t}$ values, from a 10-min data collection, with their corresponding uncertainties during 2013 maintenance operations, using the same gas passing through all VDCs operated with identical parameters.

\subsection{Performance}

\subsubsection{Gas anomaly detection}

The VDC system has shown its ability to detect quickly any gas anomaly, coming for instance from a change of the mixture or from some ambient air entering the system. The example shown in Fig. 7 occured when the flowmeters of the gas system were recalibrated and the gas mixture changed from 84.5/15.5 to 85/15 $\mathrm{Ar} / \mathrm{CO}_{2}$. Figure 7 shows the shifted $v_{d r i f t}$ value for all VDCs. If ambient air enters the system, the presence of $\mathrm{N}_{2}$ will result in a spike in the measured $v_{d r i f t}$. Figure 8 shows such an example that occured in one wheel, affecting one VDC measurement. It demonstrates that the VDC system is able to detect quickly and unambiguously the $v_{\text {drift }}$ value modifications within $1 \mathrm{~h}$ or less. 


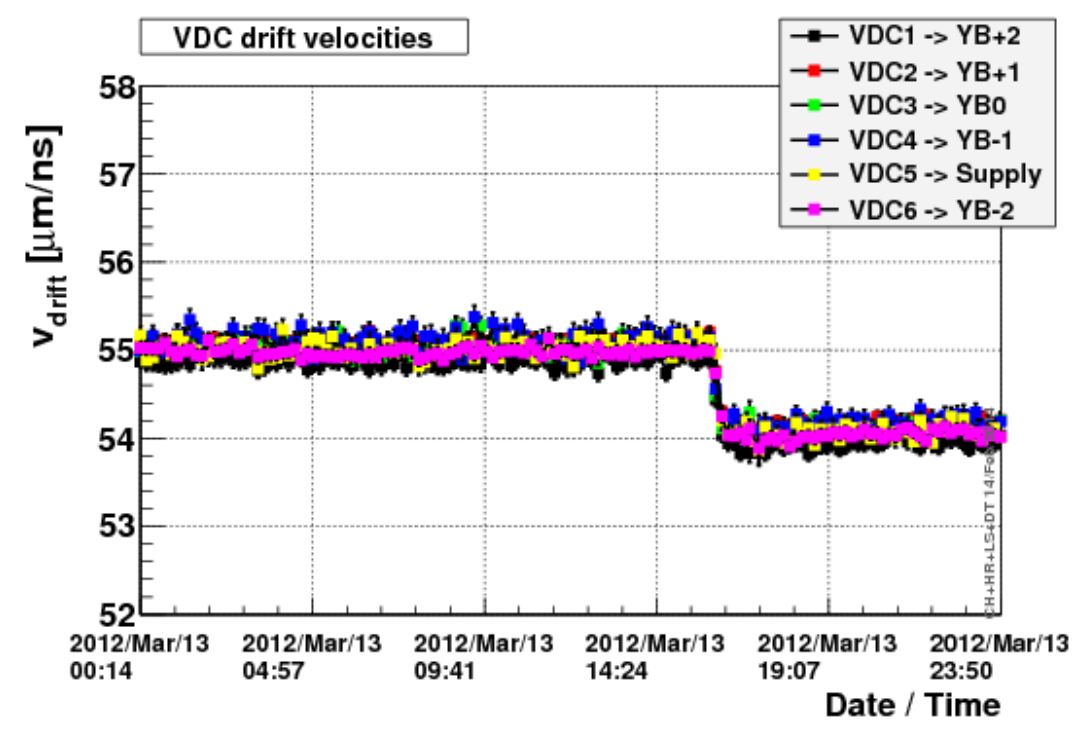

Figure 7: Example of a gas anomaly detection by the VDC system. The mixture of the gas supply changed from 84.5/15.5 to 85/15 Ar/ $/ \mathrm{CO}_{2}$. The curves from all six VDCs are superimposed. At the clearly visible step, the drift velocity changed by nearly $2 \%$. The cathode voltage was set to $-13 \mathrm{kV}$.

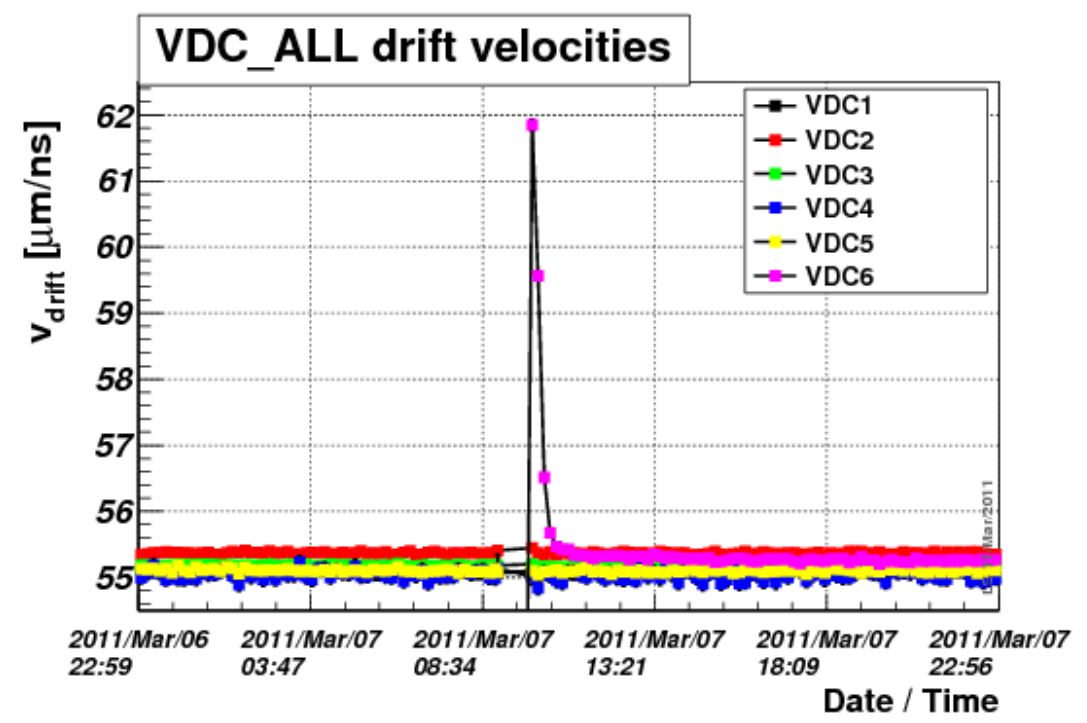

Figure 8: Example of a gas anomaly detection by the VDC system due to a contamination by ambient air in a DT after a maintenance operation, as flagged by one VDC. The curves from all six VDCs are superimposed. In the normal operation mode, the six VDCs analyze samples from different DT chambers for about $1 \mathrm{~h}$ and then switch to the next DT chamber. 


\subsubsection{Aging}

The typical aging of wire chambers, related to deposition of material on the surface of the anode wire, will also affect the VDC. Two characteristics of the VDC (Fig. 9, insert) help to visualize the mechanism and the onset of aging: a) The beam of electrons from the radioactive source has a diameter of about $6.7 \mathrm{~mm}$ at the region where its ionization products are transported to the anode wire. This implies that the large signal rate is well localized along the length of the anode wire. b) The drifting electrons reach the anode wire passing through the $2.5 \mathrm{~mm}$ wide collimator at a distance of about $10 \mathrm{~mm}$ from the anode wire, such that they all come from one side of the anode wire. These two geometrical peculiarities of the VDC make it possible to look at the relationship between the impinging drift electrons and the deposits on the wire. The photographs in Fig. 9 are of several places along an anode wire after about two years of continuous operation of the VDC. They show that there are significant deposits on the anode wire of the VDC and that they are mostly present in the region of the impinging drift electrons. Furthermore, the deposits are predominantly on the side of the wire exposed to the drift electrons. Further inspection with a raster electron microscope showed that the deposits consist essentially of $\mathrm{C}, \mathrm{O}$, and $\mathrm{Si}$.

As a consequence of these deposits on the anode wire, the VDC anode rate decreases with a time constant of less than a year. By raising the anode voltage to recover the signal amplitude, the VDCs have been operated for over more than two years. Because the VDC has only one anode wire, which can readily be exchanged as part of maintenance, the anode wire is preferably exchanged every year.

Compared with the large DT drift chambers used in the barrel region of the CMS experiment, there are differences in operational parameters that may have an impact on the aging. The VDC has about the same volume as a DT drift cell. The gas flow of $5 \mathrm{~L} / \mathrm{h}$ through the VDC is about 60 times larger than the flow through a drift cell of the DT because the total gas flow of $50 \mathrm{~L} / \mathrm{h}$ through the DT chamber is shared on average by 700 drift cells. Because the deposition on the anode is primarily related to the number of drifting electrons impinging on the surface of the anode, this rate can also be compared. For two sources of $10 \mathrm{MBq}$ activity each, the collimators at the inlets to the VDC lead to a total nominal rate inside the VDC of $4.1 \mathrm{kHz}$. Although the collimators at the VDC outlets further reduce the rate of particles reaching the trigger unit, for aging considerations it is the total anode rate inside the VDC that matters. The drifting electrons will all impinge on the anode wire surface over the restricted area described above. The hit rates in the DT drift cells are below $10 \mathrm{~Hz} / \mathrm{cm}^{2}$ for a luminosity of $10^{34} \mathrm{~cm}^{-2} \mathrm{~s}^{-1}$, which means $40 \mathrm{~Hz} / \mathrm{cm}$ on the whole surface of the anode wire of $50 \mu \mathrm{m}$ diameter. The signal rate per unit area of the anode wire surface is therefore over 6000 times larger in the VDC when compared with the largest DT drift cell rate. From this point of view, the VDC serves as an indicator for future aging of the large DT chambers.

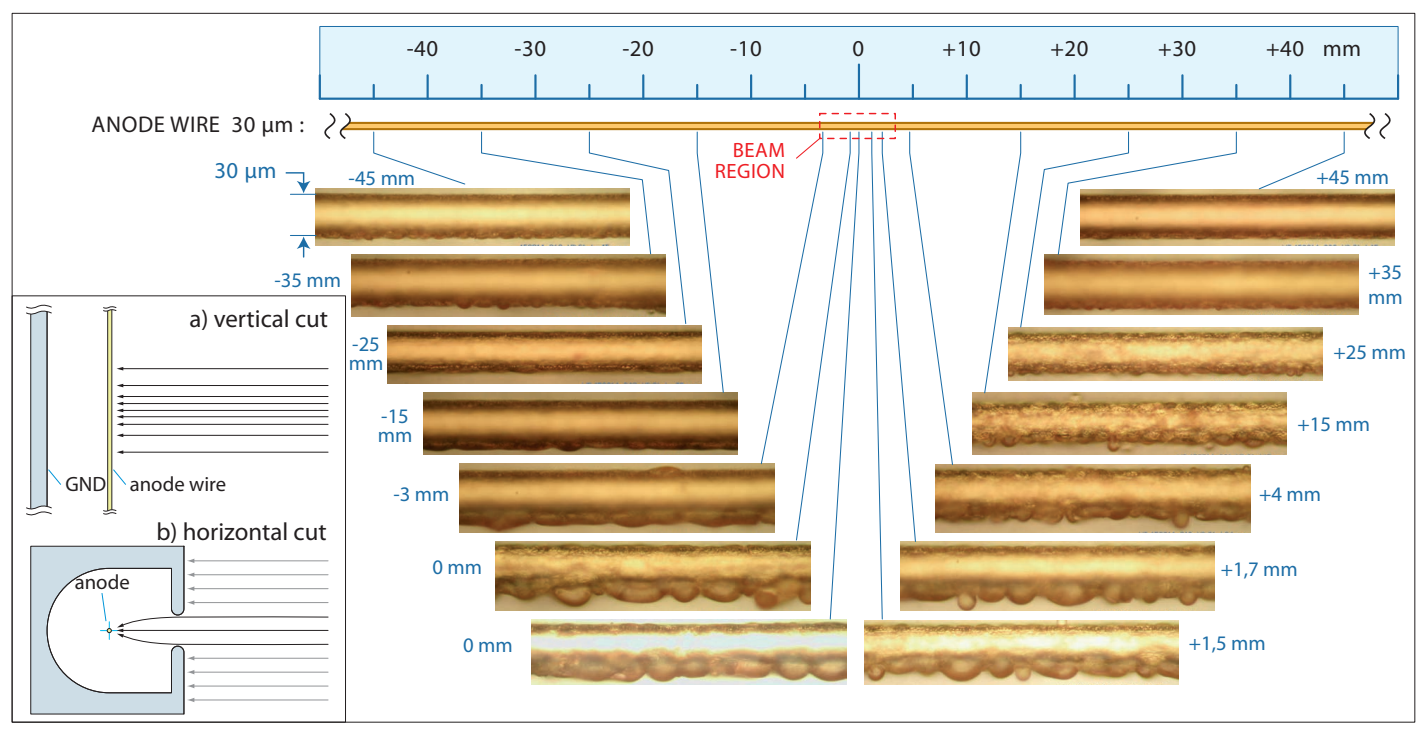

Figure 9: Microphotographs at several places along an anode wire after about two years of uninterrupted operation. In the photographs, light reflection causes the wire borders to appear dark and the center region bright. The photographs taken at a distance of $45 \mathrm{~mm}$ show a clean wire, while those taken around $0 \mathrm{~mm}$ show large deposits. Generally, the deposits on the wire are more pronounced in the region of the beam and on one side of the wire. Insert: the anode region of the VDC. The ionization electrons drift towards the anode, but only those passing the narrow collimator slit reach the anode wire. The diameter of the anode wire is not to scale. 


\subsubsection{Uncertainties on the drift velocity measurement}

In the design and operation of the VDC, effort is made to keep systematic uncertainties small, or to reduce their impact on the measured value of $v_{\text {drift }}$, as described in the following. Systematic uncertainties in the distance of $48 \mathrm{~mm}$ between the collimators for the beams from the sources directly affect the value of the $v_{\text {drift }}$ obtained. On the inlet and outlet sides, the machining of the two collimator holes is estimated to contribute a $\pm 20 \mu \mathrm{m}$ tolerance. On the outlet side the slits defining the position of the scintillating fiber are about $0.1 \mathrm{~mm}$ wider than the $1-\mathrm{mm}$ nominal width of the fiber to avoid damaging it. If the fiber is thick enough to touch the borders of the slit, this widening does not contribute to the incertitude. Thus, for a fiber exactly $1 \mathrm{~mm}$ thick, the effective distance between both fiber segments may deviate by at most two times $\pm 50 \mu \mathrm{m}$, i.e., $\pm 100 \mu \mathrm{m}$ from the nominal $48 \mathrm{~mm}$. The mechanical tolerances are upper limits; the corresponding standard deviation is about $29 \%$. These incertitudes are for the outer walls of the VDC; their impact at the center of the VDC, where the ionization electrons reaching the anode originate, is half of these values. Together, the standard deviation of the effective distance between the two beams at the center of the VDC may vary between 10 and $38 \mu \mathrm{m}$. This can induce a difference in the time of flight between both beams, corresponding to an expected systematic inaccuracy of $0.02 \%$ to $0.08 \%$ of the $v_{\text {drift }}$ measurement. This uncertainty is reflected in a scaling of the absolute value measured, but has no impact on the resolution of the VDC.

The ionizing electron beams will be slightly deflected by the E-field in the VDC, amounting to a deflection of up to $345 \mu \mathrm{m}$ before reaching the trigger box [4]. Because both beams sense the same E-field, they will experience the same deflection and there is no consequence on the $v_{\text {drift }}$ measurement itself. The effective width of the ionizing electron beams, as delimited by the $1-\mathrm{mm}$ collimators, is reflected in a spread of about $20 \mathrm{~ns}$ in the time distribution. Occasional elastic scattering in the gas will lead to a non-straight path of the ionizing electron beam and enlarge the effective beam width, hence contributing to an enlargement of the width of the time distribution peaks.

The electrons from ionization of the gas, while drifting towards the anode, will be subject to diffusion. Since the diffusion increases with the square root of the travelled distance, the diffusion will be different for electrons from the two beams, which are at distances of 14.8 and $62.8 \mathrm{~mm}$ from the anode wire, respectively. For the gas mixture and the E-field range used in the VDC, the expected difference in longitudinal diffusion between both beams is about 3.8 ns. Longitudinal diffusion will mainly affect the width of the peaks in the time distributions, while it should have a negligible impact on the peak position.

The time measurement is done by the TDC in $0.78 \mathrm{~ns}$ wide bins. Since the time measurement is obtained as the center of a gaussian distribution with a width having a standard deviation of about $20 \mathrm{~ns}$, the TDC resolution has a negligible impact on the systematic uncertainty.

Further electronic effects generated by the rest of the readout chain will not have any impact because for each VDC we are measuring the time difference between pulses transported by the same cable and treated by the same VDC preamplifier, trigger sensors, and TDC; systematic uncertainties will thus cancel.

The magnetic field inside the VDC racks does not exceed $4 \mathrm{mT}$ and is nearly orthogonal to the direction of the electrons drift velocity. The corresponding uncertainty on $v_{d r i f t}$, as obtained from simulation with Magboltz, is below $0.007 \%$ and can be neglected.

With the field electrode geometry shown in Fig. 3, the local E-field varies along the path of the drifting electrons by $\pm 0.8 \mathrm{~V} / \mathrm{cm}$ (derived using a cathode voltage of $-13 \mathrm{kV}$ ), averaging to $0 \mathrm{~V} / \mathrm{cm}$. Several parameters can influence the E-field, the first one being the accuracy of the geometry of the VDC electrodes. The construction accuracy for the positions of the field electrodes is estimated to be about $40 \mu \mathrm{m}$. A shift of $50 \mu \mathrm{m}$ in the position of a field electrode along the E-field direction would lead to a local variation of the E-field of up to $+1 \mathrm{~V} / \mathrm{cm}$ on one side and $-1 \mathrm{~V} / \mathrm{cm}$ on the opposite side [4], which represent a local inaccuracy of the E-field of about $\pm 0.06 \%$, but both of which tend to compensate each other. The resistors located between the field electrodes were varied by $27 \mathrm{k} \Omega$ [4] for a $50 \mathrm{M} \Omega$ resistor and the simulation has shown only a negligible impact on the E-field. The presence of free electrons and ions from both ionizing beams and from the gas amplification at the anode will influence the E-field. The largest effect is from the slowly traveling ions produced by the gas amplification, but their disturbance of the E-field is limited to about $10^{-5} \mathrm{~V} / \mathrm{cm}[4]$ and is thus negligible.

Pressure and temperature also affect the drift velocity, which depends on the E-field strength divided by the gas density. To quote values under same conditions of $1000 \mathrm{mbar}$ and $20^{\circ} \mathrm{C}$, we scale the E-field value accordingly. The absolute pressure is maintained stable at $1000 \mathrm{mbar}$ by using the pressure controller system. A 1 mbar precision on the absolute pressure then translates into $0.1 \%$ in the E-field. The rack temperature and the temperature of the VDC itself are known with $0.1{ }^{\circ} \mathrm{C}$ accuracy. The gas temperature can vary up to a maximum of $1{ }^{\circ} \mathrm{C}$, which 
translates into $0.4 \%$ in the E-field, when scaling with the absolute temperature. It should be noted that we account for temperature differences by scaling the E-field value; this procedure is not perfect in the range of E-field values used in the VDC. However, simulation with Magboltz shows that for a variation by $1^{\circ} \mathrm{C}$, in our E-field range between 1100 and $2350 \mathrm{~V} / \mathrm{cm}$ the drift velocity varies by less than $0.03 \%$. From computations for the primary cathode voltage of $-13 \mathrm{kV}$ (about $2000 \mathrm{~V} / \mathrm{cm}$ ) and temperatures from 10 to $40^{\circ} \mathrm{C}$, one notices that the drift velocity remains unchanged.

Finally, the precision of the E-field is also limited by the HV supply, which has a $\pm 0.3 \%$ output voltage accuracy 11). While working in the plateau region of the drift velocity as a function of the E-field, the impact of the uncertainty of the E-field and of the pressure on the measured value of the drift velocity is strongly reduced (see Fig. 1).

The statistical uncertainty of $v_{d r i f t}$, for example, for 10000 events in the time spectrum, is $0.04 \%$. There is first of all an inherent statistical uncertainty given by the fit to both peaks (Fig. 6). In addition, the ${ }^{90} \mathrm{Sr}$ sources are not identical, as they are constructed with a $20 \%$ accuracy of the activity, which can lead to a different integral of the peaks and/or a different spectrum. Finally the trigger system and the electronic readout chain (discriminator, coincidence, TDC channel) can cause some statistical differences in the time spectrum acquisition.

The overall systematic and statistical uncertainties are also studied experimentally by flushing the same gas through all VDCs. At the top of Fig. 10, the $v_{\text {drift }}$ values measured under these conditions for the six VDCs are shown. For each VDC, the measured values in 10 -min periods fluctuate with time by about $0.05 \mu \mathrm{m} / \mathrm{ns}$, i.e., by $0.1 \%$. This is compatible with the expected statistical uncertainty of $0.09 \mu \mathrm{m} / \mathrm{ns}$. The averages over $24 \mathrm{~h}$ are expected to fluctuate 12 times less but their standard deviation is calculated to be $0.09 \mu \mathrm{m} / \mathrm{ns}$, i.e., $0.2 \%$ of the average value among the six VDCs. The expected statistical uncertainty for a 24 -h average measurement is $0.008 \mu \mathrm{m} / \mathrm{ns}$. The observed differences should thus be of a systematic nature. The systematic uncertainty is reduced by a rescaling factor for each VDC, obtained by rescaling the averages per VDC to the global average of the six VDCs. A 5-h period from a preceding run is taken to obtain the scaling factors, which are then applied to the run shown here. The scaled values are shown at the bottom of Fig. 10. Now the averages over $24 \mathrm{~h}$ differ by only $0.013 \mu \mathrm{m} / \mathrm{ns}$ among the six VDCs, i.e., by $0.02 \%$, consistent with the expected statistical fluctuations of $0.008 \mu \mathrm{m} / \mathrm{ns}$ and confirming that the scaling indeed corrects a constant systematic error. Three years of operation and maintenance work have left the scaling factors unchanged, which corroborates their systematic origin.

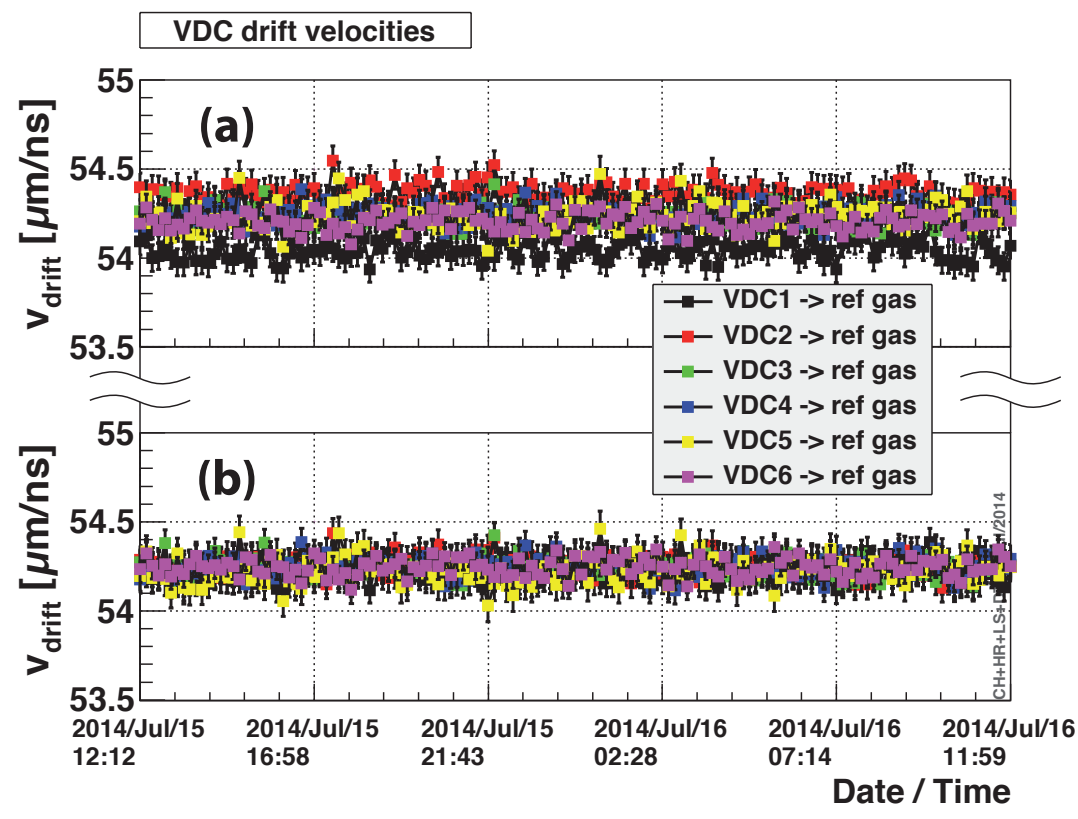

Figure 10: Systematic uncertainty study by flushing the same gas through all VDCs. Data collection 10 min per point, $-13 \mathrm{kV}$ at the cathode, and pure $85 / 15 \mathrm{Ar} / \mathrm{CO}_{2}$ gas. (a) The fluctuation of measured $v_{\text {drift }}$ values is $0.05 \mu \mathrm{m} / \mathrm{ns}$ for each VDC. (b) The $v_{\text {drift }}$ values measured by each VDC were rescaled with factors derived from the overall average of a previous run. The systematic uncertainty is reduced.

11) CAEN module A1526N : http://www.caen.it/csite/CaenProd.jsp?parent=20\&idmod=126 
Among the sources of systematic uncertainties that we discuss, the accuracy of the geometry of the VDC components is dominant. The experimental determination of the uncertainties agrees with the expectation. The overall measured uncertainty has confirmed that the statistical uncertainties are comparable to the systematic ones. The choice of measurements limited to $10 \mathrm{~min}$ is a compromise between good statistical accuracy and frequent sampling of the large muon chambers.

\section{Conclusion}

The VDC system was successfully installed at the CMS experiment in 2011, where it has been routinely sampling the gas from each of the 250 large muon DT chambers and monitoring the drift velocity with about $0.2 \%$ or $0.1 \mu \mathrm{m} / \mathrm{ns}$ statistical uncertainty. With systematic uncertainties of $0.2 \%$, the absolute value of the drift velocity is obtained with about $0.3 \%$ or $0.15 \mu \mathrm{m} / \mathrm{ns}$ precision. The achieved stability and precision of the drift velocity measurement are within the requirements. Several gas anomalies have already been revealed. The VDC system makes it possible to maintain a very high stability in the gas conditions of the CMS barrel muon chambers.

\section{Acknowledgments}

We thank our technicians and engineers F. Adamczyk, G. Fetchenhauer, H. Frohn, J. Grooten, G. Hilgers, R. Kupper, R. Lampe, B. Philipps, W. Reuter, H. Szczesny and F.P. Zantis for their skillful and patient contributions to the development of the VDC system. We acknowledge advice and help in the early phase of the project from our colleagues A. Böhm and J. Frangenheim from Aachen and G. Zilisi from Debrecen. We thank our colleagues from CERN, A. Benvenuti, R. Guida, F. Hahn, and S. Haider as well as all colleagues from the DT group and from the CMS technical coordination group for help and fruitful discussions.

This work was supported by the BMBF (Bundesministerium für Bildung und Forschung).

\section{References}

[1] The CMS Collaboration, Technical proposal, CERN/LHCC 94-038 (1994).

[2] The CMS Collaboration, The CMS experiment at the CERN LHC, JINST 3 S08004 (2008).

[3] The CMS Collaboration, The muon project, Technical design report, CERN/LHCC 97-31, CMS TDR2 (1997).

[4] G. Altenhöfer, Development of a drift chamber for drift velocity monitoring in the CMS barrel muon system, RWTH Aachen University, III. Phys. Inst. A, Diploma Thesis (2006).

[5] J. Frangenheim, Measurements of the drift velocity using a small gas chamber for monitoring of the CMS muon system, RWTH Aachen University, III. Phys. Inst. A, Diploma Thesis (2007).

[6] M. Sassowsky, Überwachung von Driftkammergasen mit einer Testkammer, RWTH Aachen University, III. Phys. Inst. A, Diploma Thesis (1990),

A. Tavenrath, Bau einer Driftkammer zur Überwachung der Gasqualität in der L3-Vertexkammer, RWTH Aachen University, III. Phys. Inst. A, Diploma Thesis (1989),

H. Anderhub et al, Experience with the L3 vertex drift chamber at LEP, Nucl. Instr. and Meth. A515(2003) 31-36.

[7] L. Baksay et al., Apparatus for precise drift time change measurements by means of photoelectrons emitted from the cathode, Nucl. Instr. and Meth. A310 (1991) 607-612.

[8] K. Fujii et al., Automated monitoring and calibrating system of gas gain and electron drift velocity: Prototype system and accumulation of reference data, Nucl. Instr. and Meth. A245 (1986) 35-44.

[9] A. Andronic et al., Drift velocity and gain in argon- and xenon-based mixtures, Nucl. Instr. and Meth. A523 (2004) 302-308.

[10] T. Zhao et al, A study of electron drift velocity in Ar-CO2 and Ar-CO2-CF4 gas mixtures, Nucl. Instr. and Meth. A340 (1994) 485-490. 
[11] M. Kollefrath, Bau einer Driftkammer zur Messung von Driftgeschwindigkeiten in Gasen, University Freiburg, Diploma Thesis (1995).

[12] S.F. Biagi, Monte Carlo simulation of electron drift and diffusion in counting gases under the influence of electric and magnetic fields, Nucl. Instr. and Meth. A421(1999) 234-240. 